\title{
Was macht eigentlich ... Richard E. Hautmann
}

\begin{abstract}
Ob in Forschung, Lehre oder Versorgung, ihre Namen sind den meisten Urologen bekannt: Persönlichkeiten wie Prof. Richard E. Hautmann haben das Fachgebiet geprägt. Auch nach dem Ruhestand sind sie häufig noch aktiv. Das zeigen die Interviews unserer neuen Serie: Was macht eigentlich ...?
\end{abstract}

Sie sind seit neun Jahren im Ruhestand. Stimmt das, oder sind Sie noch aktiv? Prof. Richard E. Hautmann: Mit 67 Jahren bin ich Ende 2009 emeritiert. In modifizierter Form betätige ich mich aber weiterhin in Forschung, Lehre und Krankenversorgung.

Haben Sie sich auf Ihren Ruhestand vorbereitet?

Hautmann: Ja, das ist aber nicht so einfach. Formal entscheidet alleine das Wissenschaftsministerium über das WIE und WO der Arbeitserlaubnis! Ein Cave sind hier die Pensionsansprüche. Ohne mein akademisches Büro inklusive meiner Chefsekretärin könnte ich derzeit nichts Wirkliches bewegen. Meine klinische Tätigkeit nach der Emeritierung findet in der Urologie des Klinikums Lüdenscheid statt. Mein ältester Sohn Stefan ist dort Klinikdirektor. Ich habe einen Vertrag als "expert consultant" (Konsiliararzt mit Liquidationsrecht). Meine Patienten kommen zu $90 \%$ aus zwei Ländern - wie auch zwei meiner "Schlüsselpatienten“: ein EG-Ministerpräsident und ein außereuropäischer König. Wissenschaftlich und akademisch bin ich noch, mit viel Reisetätigkeit, in die Amerikanische Gesellschaft für Urologie integriert, unter anderem als Ehrenmitglied und in zahlreichen Kommissionen.

Würden Sie sagen, dass Sie auf den Ruhestand gut vorbereitet waren?

Hautmann: Ja, aber unter glücklichen Voraussetzungen, wie zuvor erläutert. Eine unerwartete Erkenntnis für mich war: Nur $50 \%$ arbeiten wollen, das geht nicht!

Was vermissen Sie am meisten am aktiven Berufsleben?

Hautmann: "Vermissen“ tue ich - im umgekehrten Sinne - die sieben Jahre als Vor- standsvorsitzender des Uniklinikums Ulm und die zwei Jahre als Dekan der Medizinischen Fakultät. So wichtig diese Arbeit auch für die Institutionen war, mich haben sie viele hunderte Stunden gekostet. Die heutige Hauptamtlichkeit dieser Tätigkeit ist aber leider auch nicht besser.

\section{Würden Sie Ihre Karriere noch einmal so gestalten?}

Hautmann: Ein klares JA! Ich habe mit 18 Jahren und jugendlicher Naivität einen Flug zu den Sternen programmiert - mit Erfolg.

Was waren die Highlights Ihrer Karriere? Hautmann: In der Grundlagenforschung war das Jahr in der Pharmakologie mit der Erforschung des "renal handling of oxalate" ein Highlight. In der Klinik war die Entwicklung der Neoblase herausragend. Der Aufbau einer großen Universitätsklinik mit vielen leistungsstarken Mitarbeitern und einer exzellent arbeitenden Pflegedienstleitung zähle ich auch zu den Höhepunkten. Menschlich war dies vor allem meine Ehefrau, die "mich hat machen lassen“.

\section{Was waren Ihre Downlights?}

Hautmann: Weltweit ist die Urologie trotz idealem Zugriff auf die Entscheidungsträger, also unsere Patienten, eine Randerscheinung geblieben. Der Zustand der heutigen Medizin in Baden-Württemberg wurde entscheidend im Wissenschaftsministerium in Stuttgart geprägt. Trotz meiner Tätigkeit als Vorstandsvorsitzender konnte ich mit den Kollegen der vier anderen Unikliniken aus Baden-Württemberg nicht verhindern, dass wir Professoren als Erfüllungsgehilfen der Krankenhausträger inklusive der Ministerien geendet sind. Ein Zustand, der mich verstimmt. Ebenso geht es mir mit der

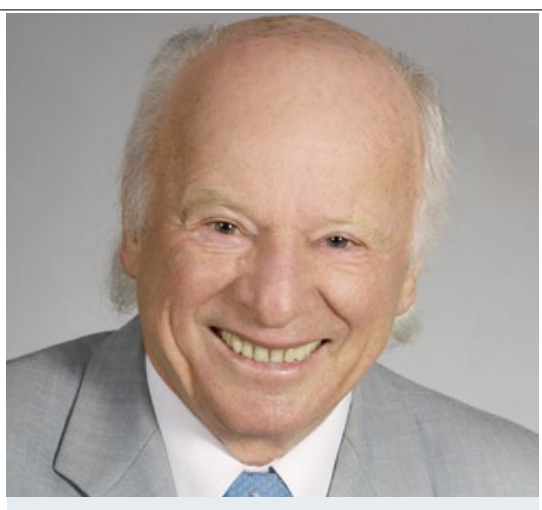

Zur Person

Prof. em. Dr. Dr. h.c. Richard E. Hautmann

Vor seiner Emeritierung leitete Richard E. Hautmann als ärztlicher Direktor 25 Jahre lang die Ulmer Universitätsklinik für Urologie und Kinderurologie. Seine medizinische Karriere begann der 1943 geborene Vater von zwei Söhnen mit dem Medizinstudium in Würzburg. Im Jahr 2013 erhielt er eine der höchsten internationalen Auszeichnungen in der Urologie, den SIU Distinguished Career Award. Aus seinen medizinischen Erfolgen ragt insbesondere die Entwicklung der "Neoblase nach Hautmann" heraus. Als Arzt galt das Interesse des in Ulm lebenden Urologen darüber hinaus dem Blasenkrebs und der Urolithiasis.

Denkweise vieler Urologen: „Bevor einer den Kopf etwas höher strecken darf, soll lieber der ganze Verein untergehen."

Was würden Sie anders machen?

Hautmann: Ich würde entweder zuerst Facharzt für Chirurgie (oder Onkologie) werden. Auch ein Jahr als verantwortlicher Arzt und Chirurg bei "Ärzte ohne Grenzen“ oder "Cap Anamur" - wie es mein jüngster Sohn Oliver gemacht hat - ist von extremem Vorteil. Ein Forschungsjahr in der theoretischen Medizin erweitert den Horizont, aber hilft der Urologie nicht wirklich.

\section{Gibt es etwas, das Sie bereuen?}

Hautmann: Nein - nicht wirklich.

Was raten Sie 60 -jährigen Kollegen?

Hautmann: Stillstand ist Rückschritt. 65 oder 67 Jahre sind heute kein Alter mehr. Alle Kollegen, auch Freunde, die gesund sind, aber aufgehört haben zu arbeiten, bauen rasch ab. Ich bin ein Sportplatzkind, nur da lernt man: Von nichts kommt nichts.

! Wir danken Ihnen für das Interview. 